\title{
Translational Research on the Mitral Valve: from Developmental Mechanisms to New Therapies
}

\author{
Daniel P. Judge $\cdot$ Roger R. Markwald • \\ Albert A. Hagège $\cdot$ Robert A. Levine
}

Received: 24 August 2011 / Accepted: 29 August 2011 /Published online: 7 September 2011

(C) Springer Science+Business Media, LLC 2011

Although the mitral valve (MV) plays a critical role in
cardiac function, preventing reversal of systolic flow
retrograde into the left atrium, it is rarely afforded the
attention received by the coronary arteries, the His-Purkinje

D. P. Judge $(\bowtie)$

Division of Cardiology,

Johns Hopkins University School of Medicine,

Ross 1049; 720 Rutland Avenue,

Baltimore, MD, USA

e-mail: djudge@jhmi.edu

D. P. Judge $\cdot$ A. A. Hagège

Université Paris Descartes, Sorbonne Paris Cité,

Paris, France

R. R. Markwald

Department of Regenerative Medicine and Cell Biology,

Medical University of South Carolina,

Charleston, SC, USA

A. A. Hagège

INSERM U633,

Paris, France

A. A. Hagège

Paris Cardiovascular Research Center,

Paris, France

A. A. Hagège

Department of Cardiology, Assistance Publique-Hôpitaux de Paris, Hôpital Européen Georges Pompidou,

Paris, France

R. A. Levine

Cardiac Ultrasound Laboratory,

Massachusetts General Hospital, Harvard Medical School,

Boston, MA, USA system, or the four distinct cardiac chambers. Yet the MV is indeed a dynamic and vital structure, influenced by its developmental origin, its various intrinsic cell types, and numerous growth factors that can alter its homeostasis in health or disease. Both acquired and inherited conditions may affect morphology, function, and stability of the MV apparatus. Today, treatment for MV disease is largely surgical, yet new insights will inevitably lead to the discovery and investigation of novel therapeutics. This issue of Journal of Cardiovascular Translational Research highlights MV development and disease from widely varied perspectives.

The normal MV apparatus consists of two leaflets, anterior and posterior, as well as the surrounding annulus, the papillary muscles, and its chordae tendineae that connect the papillary muscles to the leaflets. Each leaflet is divided into three segments according to the standard Carpentier nomenclature [1]. The saddle shape of the annulus reduces leaflet stress and optimizes function of the leaflets and associated chordae and papillary muscles [2-4]. The epidemiology of MV disease extends from common to rare. MV prolapse, which was once considered to affect as many as $15 \%$ or more of people in the population analyses, was rigorously defined and measured for its prevalence in the Framingham study as present in $2 \%$ of this cohort $[2,5]$. Although less common in developed countries, rheumatic MV disease continues to wreak a heavy toll of cardiovascular morbidity and expense in many developing nations around the world. Ischemic mitral valve insufficiency complicates $20-55 \%$ of acute myocardial infarctions, and its presence portends worsened prognosis [6].

The articles in this issue approach investigation of MV disease from a variety of perspectives. Salhiyyah, Yacoub, 
and Chester provide a general overview of the cells that are present in the MV, their relationship to various inherited and acquired diseases, and their associated vasculature and innervation. Also at the cellular level, Bischoff and Aikawa focus on a critical step in valvulogenesis and valve homeostasis: endothelial-mesenchymal transformation. They introduce an intriguing concept that valvular endothelial cells serve as a reservoir of progenitor cells that may replenish other valve cells in response to injury or disease. Moving to the whole organ from a developmental perspective, Peal, Lynch, and Milan review the many distinct advantages of using zebrafish in cardiac valve investigation. Although there are notable differences between the heart of zebrafish and humans, there are crucial similarities for which zebrafish are highly relevant and readily analyzed. Hakuna, Kimura, Yoshioka, and Fukuda review their groundbreaking work on angiogenic and angioinhibitory factors, such as periostin and chondromodulin, respectively. A highly regulated balance between these factors is required not only for normal cardiac valve development, but also for its homeostasis with aging and acquired diseases.

Three specific disorders with prominent MV pathology are also highlighted in this issue. Since the recognition that FBN1 mutations cause Marfan syndrome over 20 years ago, there has been a sea change in our understanding of its pathogenic basis [7]. The traditional thinking that a deficiency of fibrillin-1 would lead to inevitable structural collapse of affected tissues fails to adequately explain certain aspects of this disease, such as elongation and thickening of the MV. With that in mind, research during much of the past decade has evaluated a regulatory role for fibrillin-1 in maintenance of appropriate latent binding of transforming growth factor beta (TGF $\beta)[8,9]$. Accordingly, Judge, Rouf, Habashi, and Dietz review the pathogenesis of MV disease in Marfan syndrome and associated inherited disorders of TGF $\beta$ dysregulation. X-linked MVP is another rare condition that sheds important light onto MV morphogenesis and function. Investigators in the west of France astutely recognized that some men with MV disease requiring surgical repair had mild elevation in their activated partial thromboplastin time with mild deficiency of Factor VIII, encoded by a gene at chromosome Xq28 [10]. This important observation led to assembly of a unique cohort for a standard positional cloning approach that led to their discovery of FLNA mutations in a nearby but different gene at the same chromosomal locus [11]. As reviewed by Lardeux, Kyndt, Lecointe, Le Marec, Merot, Schott, Le Tourneau, and Probst, filamin A is a large protein with many interacting proteins and pathways. Not surprisingly, there is a wide spectrum of different phenotypes caused by FLNA mutations, and current investigations seek to understand its regulatory or functional role in MV development and function-as with fibrillin1 -reinforcing the theme of regulatory and developmental defects induced by mutations of structural proteins [12]. MV disease in hypertrophic cardiomyopathy is also reviewed in this issue. We have known for more than 20 years that sarcomere gene mutations cause this disorder of inappropriate cardiac hypertrophy, which is often complicated by MV pathology that is central to the pathophysiology of obstruction but of unknown origin. Hagège, Bruneval, Levine, Neamatalla, Desnos, and Judge highlight the spectrum of MV abnormalities known to occur in this condition, their consequence, and current theories regarding their cause, in which alterations in developmental pathways translate to disease of the adult MV.

By their very nature, review articles tend to look retrospectively at a body of relevant research, and as one reads these manuscripts, the gaps in our knowledge are quite notable. We are well on the way to recognizing the relevant factors that cause MV pathology, yet our treatments today remain largely surgical. The next phase of investigation will focus on translating these discoveries into targeted treatments. Funding by agencies such as the US National Institutes of Health, the European Union, and private foundations like Fondation Leducq is critical for the assembly of talented teams of investigators with complementary but varied strategies and perspectives, facilitating cross-fertilization of ideas. Looking to the future, we anticipate that these lines of investigation will lead to improved therapies, and will also facilitate the addition of MV diseases to the growing list of disorders for which personalized medicine finds inevitable application in the years ahead.

Acknowledgments Supported in part by grant 07CVD04 of the Leducq Foundation, Paris, France, for the Leducq Foundation Transatlantic Network of Excellence in Mitral Valve Disease, and by a Leducq Foundation Career Development Award (Daniel P. Judge.).

\section{References}

1. Carpentier A. (2010). In: Carpentier A., Adams D. H. Filsoufi F. (eds). Reconstructive valve surgery, vol. 1. Saunders Elsevier, Maryland Heights, MO, pp. 267.

2. Levine, R. A., Handschumacher, M. D., Sanfilippo, A. J., et al. (1989). Three-dimentional echocardiographic reconstruction of the mitral valve, with implications for the diagnosis of mitral valve prolapse. Circulation, 80(3), 589. 
3. Salgo, I. S., Gorman, J. H., 3rd, Gorman, R. C., et al. (2002). Effect of annular shape on leaflet curvature in reducing mitral leaflet stress. Circulation, 106(6), 711.

4. Jensen, M. O., Jensen, H., Levine, R. A., et al. (2011). Saddleshaped mitral valve annuloplasty rings improve leaflet coaptation geometry. The Journal of Thoracic and Cardiovascular Surgery, 142(3), 697.

5. Freed, L. A., Levy, D., Levine, R. A., et al. (1999). Prevalence and clinical outcome of mitral-valve prolapse. The New England Journal of Medicine, 341(1), 1.

6. Levine, R. A., \& Schwammenthal, E. (2005). Ischemic mitral regurgitation on the threshold of a solution: from paradoxes to unifying concepts. Circulation, 112(5), 745.

7. Dietz, H. C., Cutting, G. R., Pyeritz, R. E., et al. (1991). Marfan syndrome caused by a recurrent de novo missense mutation in the fibrillin gene. Nature, 352(6333), 337.
8. Ng, C. M., Cheng, A., Myers, L. A., et al. (2004). TGF-beta-dependent pathogenesis of mitral valve prolapse in a mouse model of Marfan syndrome. The Journal of Clinical Investigation, 114(11), 1586.

9. Habashi, J. P., Judge, D. P., Holm, T. M., et al. (2006). Losartan, an AT1 antagonist, prevents aortic aneurysm in a mouse model of Marfan syndrome. Science, 312(5770), 117.

10. Kyndt, F., Schott, J.-J., Trochu, J.-N., et al. (1998). Mapping of Xlinked myxomatous valvular dystrophy to chromosome Xq28. The American Journal of Human Genetics, 62(3), 627.

11. Kyndt, F., Gueffet, J.-P., Probst, V., et al. (2007). Mutations in the gene encoding filamin $\mathrm{A}$ as a cause for familial cardiac valvular dystrophy. Circulation, 115(1), 40.

12. Norris, R. A., Moreno-Rodriguez, R., Wessels, A., et al. (2010). Expression of the familial cardiac valvular dystrophy gene, filamin-A, during heart morphogenegis. Developmental Dynamics, 239(7), 2118. 